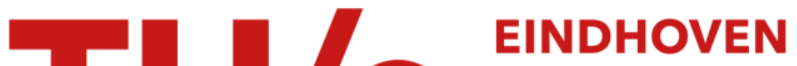 UNIVERSITY OF TECHNOLOGY
}

\section{Accurate and Efficient Evaluation of the Scattering of Bodies of Revolution Based on Magnetic Field Integral Equation}

\section{Citation for published version (APA):}

Sepehripour, F., \& van Beurden, M. C. (2022). Accurate and Efficient Evaluation of the Scattering of Bodies of Revolution Based on Magnetic Field Integral Equation. Progress in Electromagnetics Research M, 108, 1-15. [21112801]. https://doi.org/10.2528/PIERM21112801

DOI:

10.2528/PIERM21112801

Document status and date:

Published: 30/01/2022

\section{Document Version:}

Publisher's PDF, also known as Version of Record (includes final page, issue and volume numbers)

\section{Please check the document version of this publication:}

- A submitted manuscript is the version of the article upon submission and before peer-review. There can be important differences between the submitted version and the official published version of record. People interested in the research are advised to contact the author for the final version of the publication, or visit the $\mathrm{DOI}$ to the publisher's website.

- The final author version and the galley proof are versions of the publication after peer review.

- The final published version features the final layout of the paper including the volume, issue and page numbers.

Link to publication

\section{General rights}

Copyright and moral rights for the publications made accessible in the public portal are retained by the authors and/or other copyright owners and it is a condition of accessing publications that users recognise and abide by the legal requirements associated with these rights.

- Users may download and print one copy of any publication from the public portal for the purpose of private study or research.

- You may not further distribute the material or use it for any profit-making activity or commercial gain

- You may freely distribute the URL identifying the publication in the public portal.

If the publication is distributed under the terms of Article 25fa of the Dutch Copyright Act, indicated by the "Taverne" license above, please follow below link for the End User Agreement:

www.tue.nl/taverne

Take down policy

If you believe that this document breaches copyright please contact us at:

openaccess@tue.nl

providing details and we will investigate your claim. 
Progress In Electromagnetics Research M, Vol. 108, 1-15, 2022

\title{
Accurate and Efficient Evaluation of the Scattering by Bodies of Revolution Based on the Magnetic Field Integral Equation
}

\author{
Fahimeh Sepehripour* and Martijn C. van Beurden
}

\begin{abstract}
The integrals arising in magnetic field integral equation (MFIE) can become highly singular, rendering their numerical computation extremely challenging. Here, we propose a technique by which the singular integrals of the MFIE can be accurately and efficiently evaluated. In this technique, the corresponding integrals are separated into singular and regular parts. The regular parts are computed using a very simple Fast Fourier transform, whereas the remaining singular parts are evaluated based on two three-terms recurrence relations. The accuracy of the proposed method is demonstrated by analyzing the scattering of various bodies with smooth or non-smooth geometries and comparing the results with the literature.
\end{abstract}

\section{INTRODUCTION}

The analysis of scattering by perfectly electrically conducting (PEC) objects is an important problem in electromagnetics. One branch of solution methods to characterize the electromagnetic scattering is based on the electric field integral equation (EFIE) $[1,2]$. In this method, the equivalence principle is employed to obtain an integral representation for the electric fields inside or outside the object. This approach can be applied to a large variety of structures with different shapes. In [3, 4], for instance, the applicability of the EFIE approach for characterizing various kinds of PEC scatterers such as spheres and cylinders has been demonstrated. Similarly, the scattering from other kinds of scatterers like cones $[5,6]$ and cubes $[7]$ has been recently investigated leveraging the EFIE technique. Despite these findings, this method is accompanied by interior resonances that affect its accuracy when it comes to closed structures whose size is larger than the wavelength $[8,9]$. An alternative strategy to characterize the electromagnetic scattering is based on the magnetic field integral equation (MFIE), where the equivalence principle is applied to the magnetic field inside or outside of the object [10,11]. Similar to the EFIE method, this approach has also been investigated in a variety of reports in order to characterize the scattering by PEC objects. In [12], for instance, the scattering from a PEC cube and a conducting octahedron has been characterized by solving the associated magnetic field integral equations using the method of moments (MoM). Likewise, in [13], the MFIE approach was employed to analyze the scattering of PEC spheres with various sizes, and shown to provide an acceptable accuracy. Compared to the EFIE, the MFIE approach provides one with better stability and conditioning, particularly for large structures [14].

For three-dimensional scatterers, a lot of computation time and effort are required to solve the associated integral equations for both EFIE and MFIE cases. This computational burden can be significantly mitigated when the scatterer has an axially symmetric body. Such kinds of bodies, called bodies of revolutions (BOR) [5,6,15-20], allow one to reduce the computational requirement from a two-dimensional manifold to an infinite sequence of one-dimensional manifold. This is accomplished by decomposing the associated two-dimensional problem into a sequence of independent one-dimensional

\footnotetext{
Received 28 November 2021, Accepted 17 January 2022, Scheduled 30 January 2022

* Corresponding author: Fahimeh Sepehripour (f.sepehripour@tue.nl).

The authors are with the Department of Electrical Engineering, Eindhoven University of Technology, The Netherlands.
} 
problems, one for each mode in the direction of rotational invariance, thus paving the way for the accurate analysis of large objects with axial symmetry.

Currently, there are a lot of reports in the literature for scattering analysis of PEC BORs based on the EFIE approach [21-25]. Yet, little exists on the computation of such kinds of problems based on the MFIE [25-29]. In [25-27], the scattering by PEC spheres of various sizes have been analyzed based on the MFIE approach. The computational efficiency of such kinds of scattering problems was then increased in a more recent work [29], in which the associated computational burden was significantly mitigated by reducing the number of basis functions. All of these papers, however, focus on a PEC BOR with a smooth geometry, namely a sphere. On the other hand, there are also reports focusing on PEC BORs with non-smooth boundaries. Reference [28] is a prominent example, where the scattering of a PEC BOR with a non-smooth boundary, namely a PEC cylinder, has been studied using the MFIE technique. However, the accuracy of the method was found not good enough for such kind of BOR with non-smooth geometry. This is because, for non-smooth boundaries, the Green function in the integral equations becomes hypersingular when the source and observation points coincide, thus rendering the computation of the corresponding integrals difficult and often inaccurate. To overcome this vexing issue, it is crucial to develop techniques by which the singularities of the corresponding integral equation can be properly handled. While a singularity extraction method has already been proposed to address the singularity problem of the EFIE [30], its extension to the case of MFIE has not been reported to our knowledge.

Inspired by the method proposed in [30], we develop a singularity extraction technique for the MFIE for a perfectly conducting BOR. In the proposed method, we decompose the associated integrals into singular and regular parts in a way that the regular part is computed using the fast Fourier transform (FFT). To evaluate the singular parts, we develop a recurrence relation in terms of the mode index. Together, the efficient calculations of the regular and singular parts allow one to solve the associated complicated integrals accurately. The accuracy of the proposed method is investigated by analyzing the scattering from PEC bodies of revolution with smooth and non-smooth boundaries.

\section{MAGNETIC FIELD INTEGRAL EQUATION FORMULATION}

We start our analysis by considering the PEC BOR shown in Fig. 1(a). The BOR is assumed to be embedded in free-space. The BOR is assumed to be axially symmetric with respect to the $z$-axis. Leveraging this symmetry, one can define the BOR as a surface generated by a planar curve revolving around the $z$-axis. This planar curve, called the generating curve, is illustrated in Fig. 1(b) and is parameterized by $\sigma$, i.e., on the surface we have $\rho(\sigma)$ and $z(\sigma)$. An obliquely incident plane wave is assumed to impinge on the surface of the BOR. The parameters of the incident field are schematically

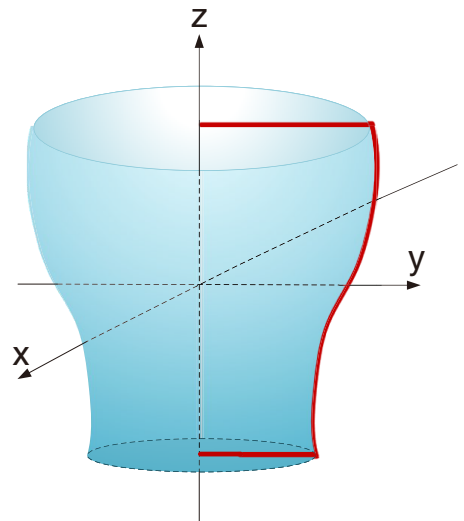

(a)

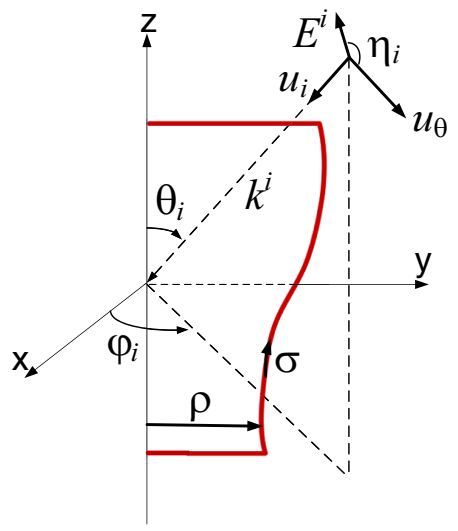

(b)

Figure 1. (a) Three dimensional sketch of the body of revolution (BOR). (b) Generating curve of the BOR and the parameters of the incident field. 
shown in Fig. 1(b). The corresponding magnetic field integral equation can be derived from the magnetic-field boundary condition across the surface of a PEC body, as, e.g., given in [17, 14, 25], i.e.,

$$
\hat{\mathbf{n}}(\mathbf{r}) \times \mathbf{H}^{i}(\mathbf{r})=\left[1-\frac{\Omega_{0}(\mathbf{r})}{4 \pi}\right] \mathbf{J}_{s}(\mathbf{r})-\hat{\mathbf{n}}(\mathbf{r}) \times P V\left(\iint \mathbf{J}_{s}\left(\mathbf{r}^{\prime}\right) \times \nabla^{\prime} G\left(\mathbf{r}, \mathbf{r}^{\prime}\right) d \mathbf{r}^{\prime}\right),
$$

in which $\mathbf{H}^{i}(\mathbf{r})$ is the incident magnetic field; $\mathbf{J}_{s}(\mathbf{r})$ is the surface current density induced by the incident field over the surface of the BOR; $\hat{\mathbf{n}}(\mathbf{r}$ ) is the (outward) unit normal vector on the surface of the BOR; $\Omega_{0}(\mathbf{r})$ is the exterior solid angle of the surface at the observation point $\mathbf{r} ; P V$ stands for the Cauchy principal value; $G\left(\mathbf{r}, \mathbf{r}^{\prime}\right)$ is the free-space Green function, defined as [25]

$$
G\left(\mathbf{r}, \mathbf{r}^{\prime}\right)=\frac{e^{-j k R}}{4 \pi R},
$$

where $k=2 \pi / \lambda$ is the free-space wave number, with $\lambda$ being the corresponding wavelength, and

$$
R=\sqrt{\rho^{2}+\rho^{\prime 2}-2 \rho \rho^{\prime} \cos \left(\phi-\phi^{\prime}\right)+\left(z-z^{\prime}\right)^{2}} .
$$

The primed and unprimed coordinates in the above equations correspond to the coordinates of the source and observation points, respectively. By taking advantage of the rotational symmetry of the BOR, the surface current density $\mathbf{J}_{s}(\mathbf{r})$ can be expanded in a Fourier series, i.e.,

$$
\mathbf{J}_{s}(\mathbf{r})=J_{\sigma}(\sigma, \phi) \mathbf{u}_{\sigma}+J_{\phi}(\sigma, \phi) \mathbf{u}_{\phi}=\sum_{m=-\infty}^{\infty}\left[J_{\sigma}^{m}(\sigma) \mathbf{u}_{\sigma}+J_{\phi}^{m}(\sigma) \mathbf{u}_{\phi}\right] e^{j m \phi} .
$$

where $J_{\sigma}^{m}$ and $J_{\phi}^{m}$ represent the modal surface current density coefficients in the $\mathbf{u}_{\sigma}$ and $\mathbf{u}_{\phi}$ directions, respectively. By employing Eq. (4), the MFIE in Eq. (1) can be split into a set of coupled equations, where the coupling appears between the two directions $\mathbf{u}_{\sigma}$ (tangential to the generating curve) and $\mathbf{u}_{\phi}$

$$
\begin{aligned}
& H_{\sigma}^{i}=-\left[1-\frac{\Omega_{0}(\mathbf{r})}{4 \pi}\right] J_{\phi}(\sigma, \phi)+\frac{1}{4 \pi} P V\left(\iint\left[\Lambda_{\sigma \sigma^{\prime}} J_{\sigma}\left(\sigma^{\prime}, \phi^{\prime}\right)+\Lambda_{\sigma \phi^{\prime}} J_{\phi}\left(\sigma^{\prime}, \phi^{\prime}\right)\right] G_{H} d \mathbf{r}^{\prime}\right), \\
& H_{\phi}^{i}=\left[1-\frac{\Omega_{0}(\mathbf{r})}{4 \pi}\right] J_{\sigma}(\sigma, \phi)-\frac{1}{4 \pi} P V\left(\iint\left[\Lambda_{\phi \sigma^{\prime}} J_{\sigma}\left(\sigma^{\prime}, \phi^{\prime}\right)+\Lambda_{\phi \phi^{\prime}} J_{\phi}\left(\sigma^{\prime}, \phi^{\prime}\right)\right] G_{H} d \mathbf{r}^{\prime}\right),
\end{aligned}
$$

in which

$$
\Lambda_{i j^{\prime}}=\mathbf{u}_{i} \cdot\left[\mathbf{u}_{j}^{\prime} \times\left(\mathbf{r}-\mathbf{r}^{\prime}\right)\right]
$$

where $i$ and $j$ can be either $\sigma$ or $\phi$, and

$$
G_{H}\left(\sigma, \sigma^{\prime}, \phi-\phi^{\prime}\right)=[1+j k R] \frac{e^{-j k R}}{R^{3}} .
$$

Note that, for the sake of simplicity, we have suppressed the $\sigma$ and $\phi$ dependency of $G_{H}$ in Eq. (5). The integrands in Eq. (5) can be further simplified by noting that $\Lambda_{i j^{\prime}}$ is a function of $\phi-\phi^{\prime}$, and consequently a periodic convolution occurs along the $\phi$ direction. By integrating both sides of each equation as $\int_{0}^{2 \pi}(.) e^{-j m^{\prime} \phi} d \phi$, one reaches a matrix equation, one for each Fourier mode $m$, of the form

$$
\left[\begin{array}{cc}
Z_{\sigma \sigma}^{m} & Z_{\sigma \phi}^{m}-\pi \\
Z_{\phi \sigma}^{m}+\pi & Z_{\phi \phi}^{m}
\end{array}\right]\left[\begin{array}{c}
J_{\sigma}^{m} \\
J_{\phi}^{m}
\end{array}\right]=\left[\begin{array}{c}
V_{\sigma}^{m} \\
V_{\phi}^{m}
\end{array}\right]
$$

in which we have assumed the exterior solid angle to be $\Omega_{0}(\mathbf{r})=2 \pi$, which holds for all observation points except for isolated points at a corner. Note that the appearance of $\pi$ on the anti-diagonal matrix blocks is due to the term containing the solid angle and the fact that the integral over $\phi$ was not normalized by $2 \pi$. Further, $V_{\sigma, \phi}^{m}=\int_{0}^{2 \pi} H_{\sigma, \phi}^{i} e^{-j m \phi} d \phi$. The corresponding (impedance) matrix elements 
are of the form

$$
\begin{aligned}
& Z_{\sigma \sigma}^{m}=j \int_{\sigma^{\prime}} \rho^{\prime}\left\{\rho^{\prime} \sin \nu \cos \nu^{\prime}-\rho \sin \nu^{\prime} \cos \nu-\left(z^{\prime}-z\right) \sin \nu \sin \nu^{\prime}\right\} S_{m} d \sigma^{\prime}, \\
& Z_{\sigma \phi}^{m}=\int_{\sigma^{\prime}} \rho^{\prime}\left\{\left[\left(\rho^{\prime}-\rho\right) \cos \nu-\left(z^{\prime}-z\right) \sin \nu\right] C_{m}+\rho^{\prime} \cos \nu B_{m}\right\} d \sigma^{\prime} \\
& Z_{\phi \sigma}^{m}=-\int_{\sigma^{\prime}} \rho^{\prime}\left\{\left[\left(\rho^{\prime}-\rho\right) \cos \nu^{\prime}-\left(z^{\prime}-z\right) \sin \nu^{\prime}\right] C_{m}-\rho \cos \nu^{\prime} B_{m}\right\} d \sigma^{\prime}, \\
& Z_{\phi \phi}^{m}=-j \int_{\sigma^{\prime}} \rho^{\prime}\left(z^{\prime}-z\right) S_{m} d \sigma^{\prime} .
\end{aligned}
$$

where $\nu$ is the angle between the tangent to the generating curve $\mathbf{u}_{\sigma}$ and the $z$-axis. This angle is defined to be positive if $\mathbf{u}_{\sigma}$ points away from the $z$-axis and negative if $\mathbf{u}_{\sigma}$ points towards the $z$-axis. In addition, we have

$$
\begin{aligned}
& B_{m}\left(\sigma, \sigma^{\prime}\right)=2 \int_{0}^{\pi} \sin ^{2}(\alpha / 2) \cos (m \alpha) G_{H}(\alpha) d \alpha, \\
& C_{m}\left(\sigma, \sigma^{\prime}\right)=\int_{0}^{\pi} \cos (\alpha) \cos (m \alpha) G_{H}(\alpha) d \alpha, \\
& S_{m}\left(\sigma, \sigma^{\prime}\right)=\int_{0}^{\pi} \sin (\alpha) \sin (m \alpha) G_{H}(\alpha) d \alpha .
\end{aligned}
$$

where $\alpha=\phi-\phi^{\prime}$. The integrals $B_{m}, C_{m}$, and $S_{m}$ can be rewritten as

$$
\begin{aligned}
C_{m} & =\frac{1}{2}\left(G_{m+1}+G_{m-1}\right), \\
B_{m} & =G_{m}-\frac{1}{2}\left(G_{m+1}+G_{m-1}\right), \\
S_{m} & =\frac{1}{2}\left(G_{m-1}-G_{m+1}\right),
\end{aligned}
$$

in which $G_{m}$ is what we call the MFIE modal Green function (MGF), defined as

$$
\begin{aligned}
G_{m}\left(\sigma, \sigma^{\prime}\right) & =\int_{0}^{\pi} \cos (m \alpha) G_{H}(\alpha) d \alpha \\
& =\int_{0}^{\pi} \cos (m \alpha)\left[1+j k R\left(\sigma, \sigma^{\prime}, \alpha\right)\right] \frac{e^{-j k R\left(\sigma, \sigma^{\prime}, \alpha\right)}}{R^{3}\left(\sigma, \sigma^{\prime}, \alpha\right)} d \alpha .
\end{aligned}
$$

\section{CONSTRUCTION OF THE RECURRENCE RELATION FOR THE MGF}

\subsection{Singularity Extraction Method}

Here, we discuss how to evaluate the MFIE modal Green function numerically. If the source and observation points coincide, the integral in Eq. (12) becomes highly singular and should be evaluated more accurately. Furthermore, the MGF is a function of the Fourier-mode index $m$, implying that it has to be computed for every single Fourier mode individually. This makes the computation of MGF extremely challenging for large bodies, for which a large number of Fourier modes are required to be considered to guarantee accuracy. Here, inspired by the method proposed in [30], we overcome this issue. To this end, we rewrite the MGF in Eq. (12) as

$$
\begin{aligned}
G_{m}\left(\rho, \rho^{\prime}, z, z^{\prime}\right) & =\int_{0}^{\pi} \cos (m \alpha)(1+j k R) \frac{(1-j k R)}{R^{3}} d \alpha+\int_{0}^{\pi} \cos (m \alpha)(1+j k R) \frac{e^{-j k R}-(1-j k R)}{R^{3}} d \alpha \\
& =G_{m}^{(1)}+G_{m}^{(2)} .
\end{aligned}
$$


Note that $R$ is a function of the integration variable $\alpha$. The second integral in Eq. (13) is a regular integral (due to the term $1+j k R$ ), which can be computed very efficiently for all modes $m$ at once using a Fast Fourier transform (FFT) [30]. Since the kernel is periodic and continuous, the associated FFT converges exponentially [31]. $G_{m}^{(1)}$, on the other hand, is associated with the singular part of the MGF. Let us focus on the evaluation of the singular part of the kernel $G_{m}^{(1)}$. After straightforward calculations, $G_{m}^{(1)}$ can be rewritten as

$$
G_{m}^{(1)}=\int_{0}^{\pi} \cos (m \alpha) \frac{1+k^{2} R^{2}}{R^{3}} d \alpha=\int_{0}^{\pi} \frac{\cos (m \alpha)}{R^{3}} d \alpha+k^{2} \int_{0}^{\pi} \frac{\cos (m \alpha)}{R} d \alpha .
$$

By defining a the traditional variable $w(0 \leq w \leq 1)$ as

$$
w=\frac{4 \rho \rho^{\prime}}{\left(\rho+\rho^{\prime}\right)^{2}+\left(z-z^{\prime}\right)^{2}} .
$$

we can rewrite Eq. (14) as

$$
\begin{aligned}
G_{m}^{(1)} & =(-1)^{m} \sqrt{\frac{w}{\rho \rho^{\prime}}}\left[\frac{1}{4} \frac{w}{\rho \rho^{\prime}} \int_{0}^{\pi / 2} \frac{\cos (2 m \alpha)}{\left(1-w \sin ^{2} \alpha\right)^{\frac{3}{2}}} d \alpha+k^{2} \int_{0}^{\pi / 2} \frac{\cos (2 m \alpha)}{\sqrt{\left(1-w \sin ^{2} \alpha\right)}} d \alpha\right] \\
& =(-1)^{m} \sqrt{\frac{w}{\rho \rho^{\prime}}}\left[\frac{1}{4} \frac{w}{\rho \rho^{\prime}} I_{m}^{(3)}+k^{2} I_{m}^{(1)}\right] .
\end{aligned}
$$

The sequence of integrals $I_{m}^{(1)}(w)$ and $I_{m}^{(3)}(w)$ satisfy two different three-terms recurrence relations, based on which we can evaluate them in $O(M)$ steps where $M$ indicates the number of truncated modes in Eq. (4) [30].

$$
\begin{aligned}
& I_{0}^{(1)}=K(\sqrt{w}), \\
& I_{1}^{(1)}=\frac{2 E(\sqrt{w})+(w-2) K(\sqrt{w})}{w}, \\
& I_{m+1}^{(1)}=\frac{1}{2 m+1}\left[4 m \frac{(w-2)}{w} I_{m}^{(1)}-(2 m-1) I_{m-1}^{(1)}\right], \\
& I_{m-1}^{(1)}=\frac{1}{2 m-1}\left[4 m \frac{(w-2)}{w} I_{m}^{(1)}-(2 m+1) I_{m+1}^{(1)}\right] .
\end{aligned}
$$

Likewise, the recurrence relation for $I_{m}^{(3)}(w)$ is

$$
\begin{aligned}
& I_{0}^{(3)}(w)=\frac{E(\sqrt{w})}{1-w}, \\
& I_{1}^{(3)}(w)=\frac{E(\sqrt{w})}{1-w}-\frac{2 E(\sqrt{w})}{(1-w) w}+\frac{2 K(\sqrt{w})}{w}, \\
& I_{m+1}^{(3)}=\frac{1}{2 m-1}\left[4 m \frac{(w-2)}{w} I_{m}^{(3)}-(2 m+1) I_{m-1}^{(3)}\right], \\
& I_{m-1}^{(3)}=\frac{1}{2 m+1}\left[4 m \frac{(w-2)}{w} I_{m}^{(3)}-(2 m-1) I_{m+1}^{(3)}\right] .
\end{aligned}
$$

in which $K(\sqrt{w})$ and $E(\sqrt{w})$ are the first and second kind complete elliptic integrals, respectively [32]. The third relations in Eqs. (17) and and (18) are the forward recurrence relations, while the fourth relations correspond to the backward case. It can be shown that, for $w \leq 0.99$, the backward recurrence relation is more accurate than the forward one. For $w \geq 0.99$, on the other hand, the forward recurrence relation is more efficient. To demonstrate this argument, we study the relative error of the forward and backward recurrence relations of $I_{m}^{(3)}(w)$ for different values of the parameter $w$. Fig. 2(a) shows the relative error of the computation of $I_{m}^{(3)}(w)$ using forward recurrence relation for different values of 


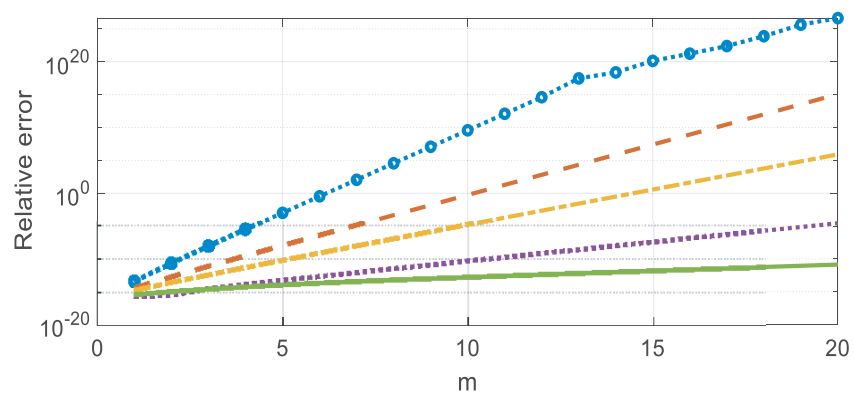

(a)

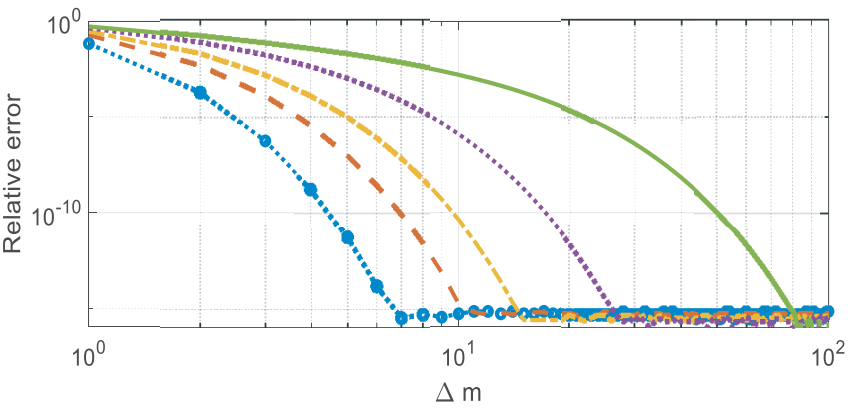

Figure 2. Relative error of $I_{m}^{(3)}(w)$, for different values of $w$, namely $w=0.2,0.5,0.7,0.9,0.99$. (a) Corresponding to the forward recurrence relation. (b) Corresponding to the backward recurrence relation, as a function of $\Delta m$.

$w$. As observed, when $w$ approaches one, the corresponding relative error becomes very small. As a result, for $w$ close to one $(w>0.99)$, we used the forward recurrence relation given in the third line of Eq. (18). Fig. 2(b) illustrates the relative error of $I_{m}^{(3)}(w)$ corresponding to the backward recurrence relation. Note that, in this figure, the value of $m$ is fixed to $m=20$. The $x$-axis in this figure represents the starting point $I_{m+\Delta m}^{(3)}(w)$ of the backward recurrence relation. As seen in this figure, for $w$ close to one, the starting point of the backward recurrence relation should be chosen far enough from the values of $m$, which are of interest. As such, for $w<0.99$, we use the backward recurrence relation provided in the fourth line of Eq. (18). Note that while we based our analysis on $I_{m}^{(3)}(w)$, similar results can be achieved for $I_{m}^{(1)}(w)$, as demonstrated in [30]. Note further that, for the results of these two figures, we have employed Mathematica's NIntegrate [33] method for numerical integration as a reference.

\subsection{Singular Nature of the Integrals $I_{m}^{(1)}(w)$ and $I_{m}^{(3)}(w)$}

Thus far, we have investigated the recurrence relations corresponding to the singular part of the Green function. Yet, the recurrence relations $I_{m}^{(1)}(w)$ and $I_{m}^{(3)}(w)$, themselves, are singular when $w=1$, which corresponds to the case where the source and observation points coincide. In particular, $I_{0}^{(1)}(w)$ and $I_{1}^{(3)}(w)$ in Eqs. (17) and (18) are singular due to the presence of $K(\sqrt{w})$, which is the first kind elliptic integral. Likewise, $I_{0}^{(3)}(w)$ exhibits a singularity due to $1-w$ in the denominator. Our next goal is to extract the aforementioned singular parts of the recurrence relations. To this aim, we replace $\cos (2 m \alpha)$ in Eq. (16) with its power series expansion of the multiple-angle cosine [22], i.e.,

$$
\cos (2 m \alpha)=\sum_{k=0}^{m} \psi_{m, k}(\cos \alpha)^{2(m-k)},
$$

where for $m \in \mathbb{N} \backslash\{0\}$, we have

$$
\psi_{m, k}=\frac{2 m}{2 m-k}\left(\begin{array}{c}
2 m-k \\
k
\end{array}\right)(-1)^{k} 2^{2(m-k)-1} .
$$

By substituting Eq. (19) in Eq. (16), the terms for which the power of $\cos (\alpha)$ is negative become singular when $w$ approaches one. We extract these terms according to the following equations

$$
\begin{aligned}
& \tilde{I}_{m}^{(1)}(w)=I_{m}^{(1)}(w)-(-1)^{m} K(\sqrt{w}) . \\
& \tilde{I}_{m}^{(3)}(w)=I_{m}^{(3)}(w)-(-1)^{m}\left[\frac{E(\sqrt{w})}{1-w}-2 m^{2} K(\sqrt{w})\right] .
\end{aligned}
$$


in which $\tilde{I}_{m}^{(1)}(w)$ and $\tilde{I}_{m}^{(3)}(w)$ are regular. The term $K(\sqrt{w})$ in Eqs. $(21)$ and $(22)$ can be represented according to the following relations

$$
\begin{aligned}
K(\sqrt{w}) \approx \ln \left(\frac{16}{1-w}\right)= & \ln \left(16\left(\left(\rho+\rho^{\prime}\right)^{2}+\left(z-z^{\prime}\right)^{2}\right)\right) \\
& -\ln \left(\left(\rho-\rho^{\prime}\right)^{2}+\left(z-z^{\prime}\right)^{2}\right) .
\end{aligned}
$$

The first term in Eq. (23) is not singular except at the axis of the rotation. As such, it can be directly computed. In order to calculate the second term in Eq. (23), we introduce new variables $l$ and $l^{\prime}$ as below

$$
\begin{aligned}
\rho & =\rho_{p}+l \Delta_{p} \sin \nu_{p}, \\
\rho^{\prime} & =\rho_{p}+l^{\prime} \Delta_{p} \sin \nu_{p}, \\
z & =z_{p}+l \Delta_{p} \cos \nu_{p}, \\
z^{\prime} & =z_{p}+l^{\prime} \Delta_{p} \cos \nu_{p} .
\end{aligned}
$$

in which $z_{p}$ and $\rho_{p}$ correspond to the starting point of the segment $p$, and $\Delta_{p}$ is associated with the width of the segment $p . \nu_{p}$ is the angle between the $z$-axis and tangent to the generating curve. By replacing the parameters defined in Eq. (24) into Eq. (23), one obtains

$$
\int_{l_{1}}^{l_{2}} \ln \left|l-l^{\prime}\right| d l^{\prime}=\left(l_{2}-l_{1}\right)-\left(l_{2}-l\right) \ln \left(l_{2}-l\right)-\left(l-l_{1}\right) \ln \left(l-l_{1}\right) .
$$

which completes the process of extracting the singularity of the modal Green function. The block diagram shown in Fig. 3 summarizes the procedure proposed to remove the singularity of the MGF of the MFIE. To verify the correctness of the proposed method further, we computed the complete integral $G_{m}$ in Eq. (12), for $m=9$, using the proposed approach and compared the result with the one obtained from Mathematica's NIntegate [33]. Fig. 4 presents the result for $0 \leq w<1, k=2 \pi$, and $\rho=\rho^{\prime}=0.25$. In this figure, the absolute values of the real and imaginary parts of $G_{m}$ are plotted in panels (a) and (b), respectively. As can be observed, the results obtained in both ways agree very well with a maximum absolute deviation of $1.7 E-10$, thus providing further evidence for the correctness of the proposed method. For validation purposes, it is worth mentioning that for the parameters specified and for $0.06<w<1$, the real part of $G_{m}$ is positive, and the imaginary part of $G_{m}$ is negative.

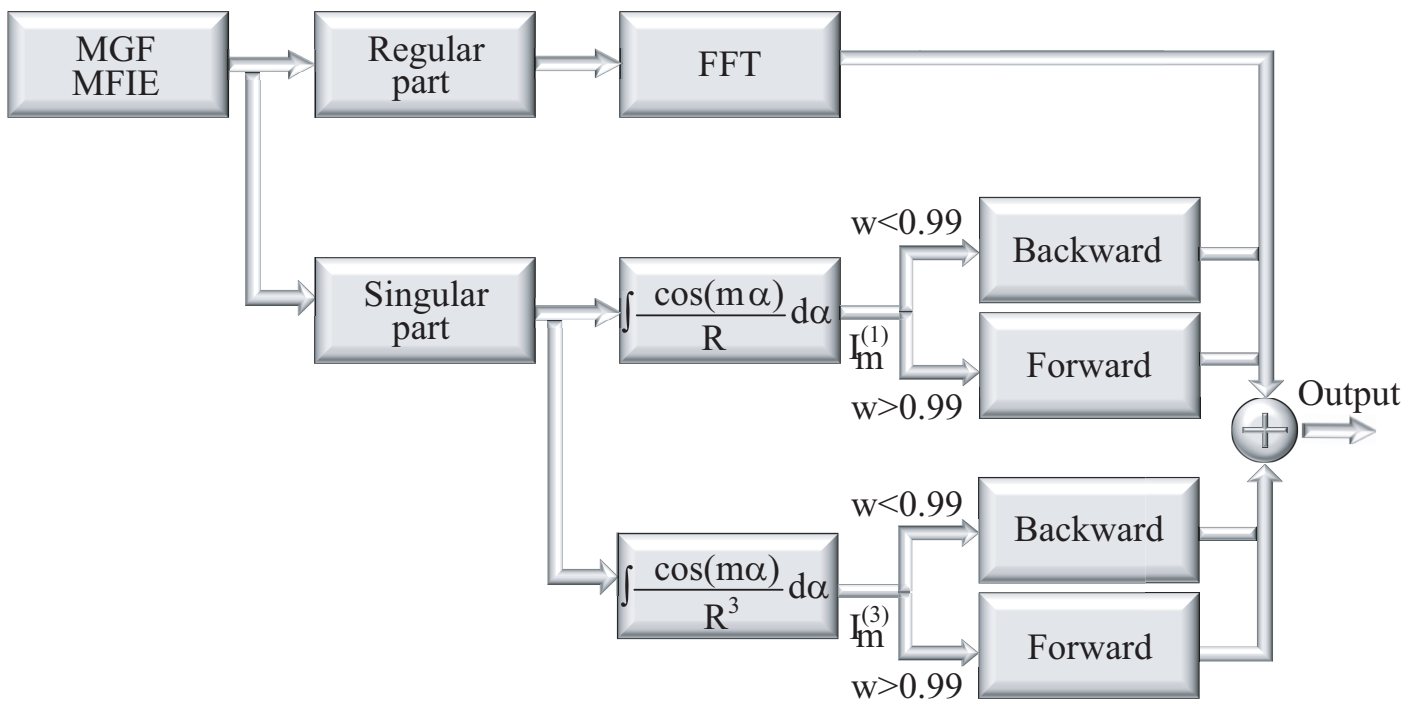

Figure 3. Conceptual block diagram of the proposed singularity extraction method. 


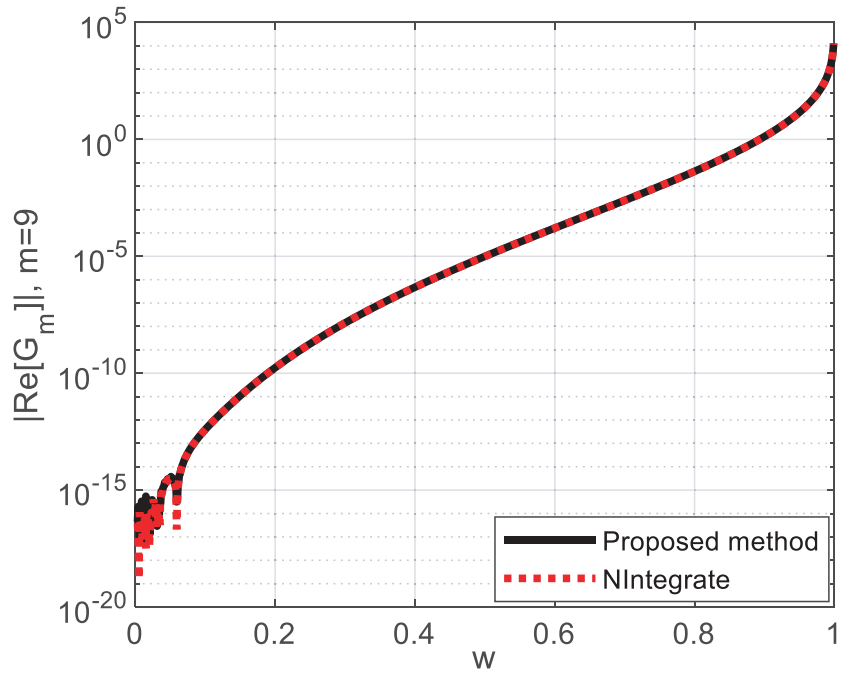

(a)

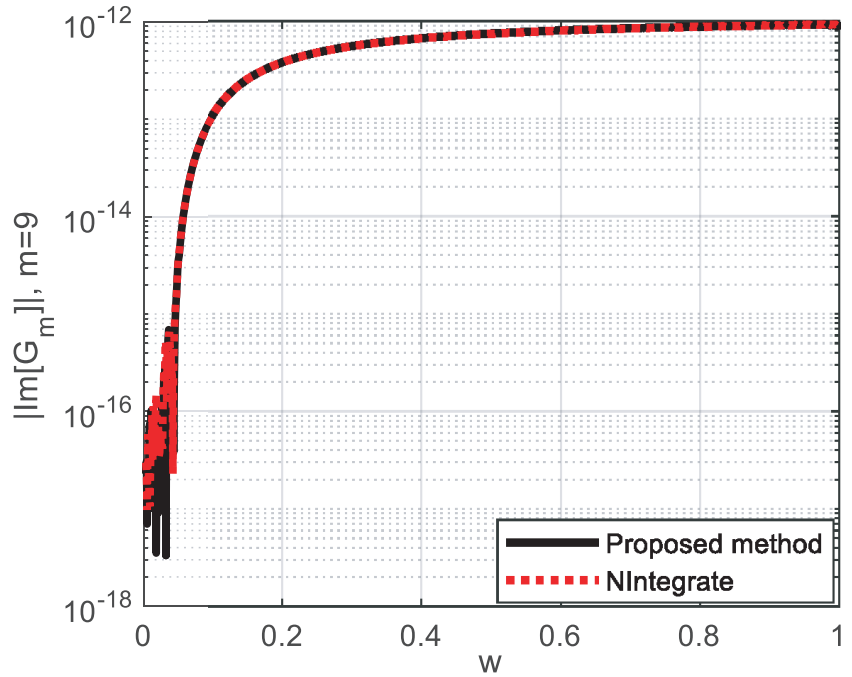

(b)

Figure 4. Absolute values of the (a) real and (b) imaginary parts of $G_{m}$ for $m=9$. The results are calculated based on the proposed method (solid black) and compared with Mathematica's NIntegrate method (dashed red).

\section{NUMERICAL RESULTS}

In order to solve the MFIE in Eq. (1) using MoM [34,25], we expand the surface current density $\mathbf{J}_{s}(\mathbf{r})$ defined in Eq. (4) on a series of basis functions, as follows

$$
\begin{aligned}
& J_{\sigma}^{m}(\sigma)=\sum_{q} I_{q m}^{\sigma} f_{q}^{\sigma}(\sigma) \\
& J_{\phi}^{m}(\sigma)=\sum_{q} I_{q m}^{\phi} f_{q}^{\phi}(\sigma)
\end{aligned}
$$

in which $I_{q m}^{\sigma}$ and $I_{q m}^{\phi}$ are the unknowns. $f_{q}^{\sigma}(\sigma)$ and $f_{q}^{\phi}(\sigma)$ are chosen to be piece-wise constant. Note that in the $\phi$ direction, the current is expanded using a finite Fourier series that is truncated from $-M$ to $M$, enabling a reasonable computational time.

We employed the so-called Galerkin method [35] to solve Eq. (8). In order to verify the accuracy of the proposed method, we analyze the scattering of electromagnetic plane waves by PEC objects with different shapes using the MFIE and then compare the results with those obtained with the EFIE. To this end, we investigate the scattering from a PEC sphere with radius $0.3 \lambda$ in free space, in which $\lambda$ is the free-space wavelength. The PEC sphere is illuminated by a $\theta$-polarized incident plane wave with angle of incidence $\theta_{i}=\pi / 4$ and $\phi_{i}=0$. Figs. 5(a) and (b) represent the induced surface current, corresponding to $m=1$, in the tangential $\left(I_{\sigma}=2 \pi \rho J_{\sigma}(\sigma, \phi)\right.$, panel a) and $\phi$ direction $\left(I_{\phi}=2 \pi \rho J_{\phi}(\sigma, \phi)\right.$, panel b). The black curves in these figures are obtained using the technique proposed here. The red lines, on the other hand, correspond to the surface current calculated using the method proposed in [36] for the EFIE. It is observed that the results match each other. We also investigated the currents over the BOR for another distinctive mode $(m=2)$. The associated results are illustrated in Figs. 5(c) and (d). Interestingly, the proposed technique also enables achieving accurate results for the case of higher-order modes. Figs. 6(a) and (c) represent the calculated tangential surface current for two specific higher-order modes, namely $m=9$ and $m=10$. Figs. 6(b) and (d) represent the associated surface currents in the $\phi$ direction for the same modes. The results of these figures verify the accuracy of the proposed method for calculating the surface currents of the higher-order modes as well. Once the currents of all modes have been calculated, the associated total current can be readily obtained from Eq. (26). The corresponding tangential and $\phi$-directed total currents are plotted in Figs. 7(a) and (b), and compared with the EFIE. 


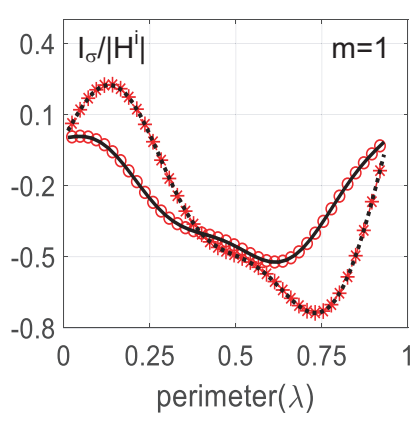

(a)

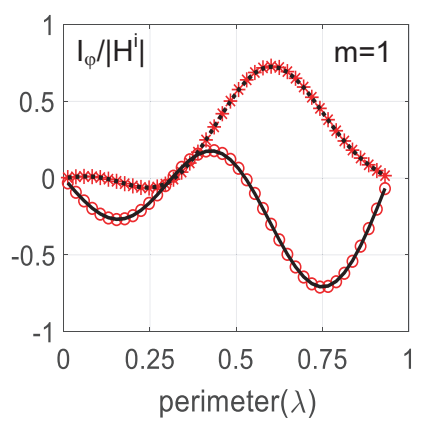

(b)

- - Re-EFIE …*...Im-EFIE

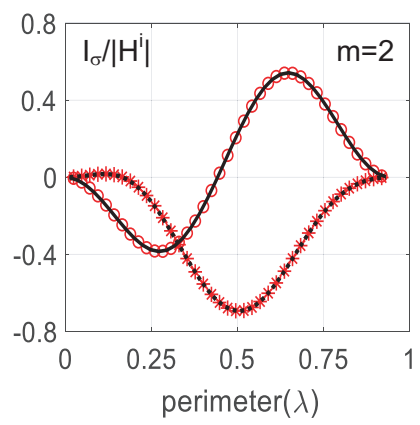

(c)

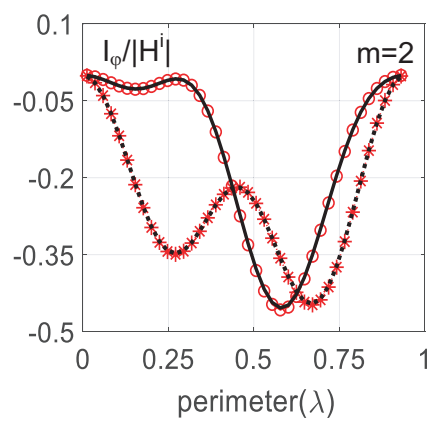

(d)

Figure 5. Surface currents (normalized to the incident magnetic field) obtained from the MFIE (black), compared with the ones of the EFIE (red). The results are reported for a perfectly conducting sphere of radius $r=0.3 \lambda$, for a $\theta$-polarized incident field. (a), (b) Tangential and $\phi$-directed current for the mode $m=1$. (c), (d) Same as (a) and (b) except that the results are reported for the mode $m=2$.

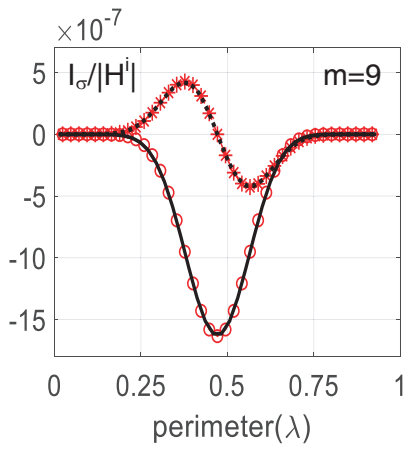

(a)

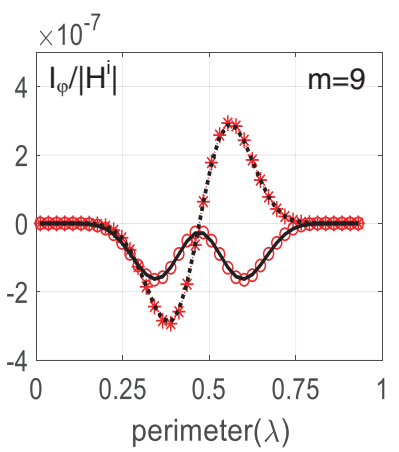

(b)

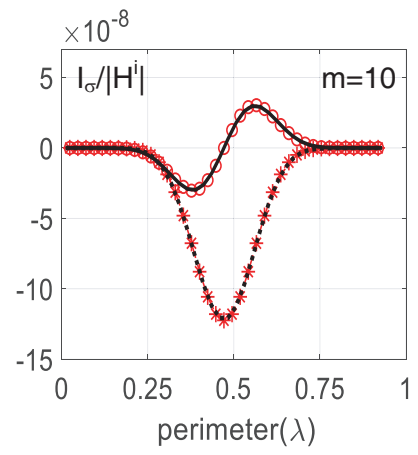

(c)

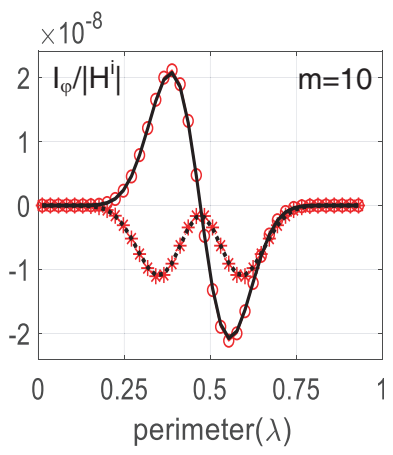

(d)

Figure 6. Surface currents obtained from the MFIE (black), compared with the ones of the EFIE (red). The results are reported for a conducting sphere possessing the radius of $r=0.3 \lambda$, for a $\theta$-polarized incident field. (a), (b) Tangential and $\phi$-directed current for the mode $m=9$. (c), (d) Same as (a) and (b) except that the results are reported for the mode $m=10$.

To characterize the proposed method for the case of bodies of revolutions possessing a non-smooth geometry, we also investigate the scattering from a PEC cylinder. We assume the radius and height of the PEC cylinder to be $r=0.25 \lambda$ and $h=0.5 \lambda$, respectively. We further assume that the cylinder is impinged by a $\theta$-polarized incident plane wave with angles of incidence $\theta_{i}=\pi / 4$ and $\phi_{i}=0$. The associated tangential and $\phi$-directed currents are shown in Fig. 8 for $m=1$ (panels (a) and (b)) and $m=2$ (panels (c) and (d)). It is observed that the results obtained using the proposed MFIE method are in general in good agreement with that of the EFIE (the maximum absolute deviation is smaller than 0.17). The tangential and $\phi$ directed currents of two higher-order modes $(m=9$ and $m=10)$ are plotted in Fig. 9. We note that, since the geometry of the BOR under investigation includes sharp edges, the agreement between the EFIE and MFIE approaches is not perfect near the corners (which correspond to 0.25 and 0.75 on the horizontal axis), especially for the currents in $\phi$ direction. To illustrate this point better, the absolute deviation corresponding to the $\phi$-directed currents is plotted in Fig. 10 for $m=1,2,9$, and 10. As observed, the deviation is larger close to the corners than the currents on the other parts of generating curve. Yet, the singularity extraction method proposed here provides much better accuracy than the situation when the singularity is not extracted and treated separately. To illustrate this, Fig. 11 shows the current densities, corresponding to the case in Fig. 9, when the singularity extraction method is not applied in the numerical computation. In the latter case, 


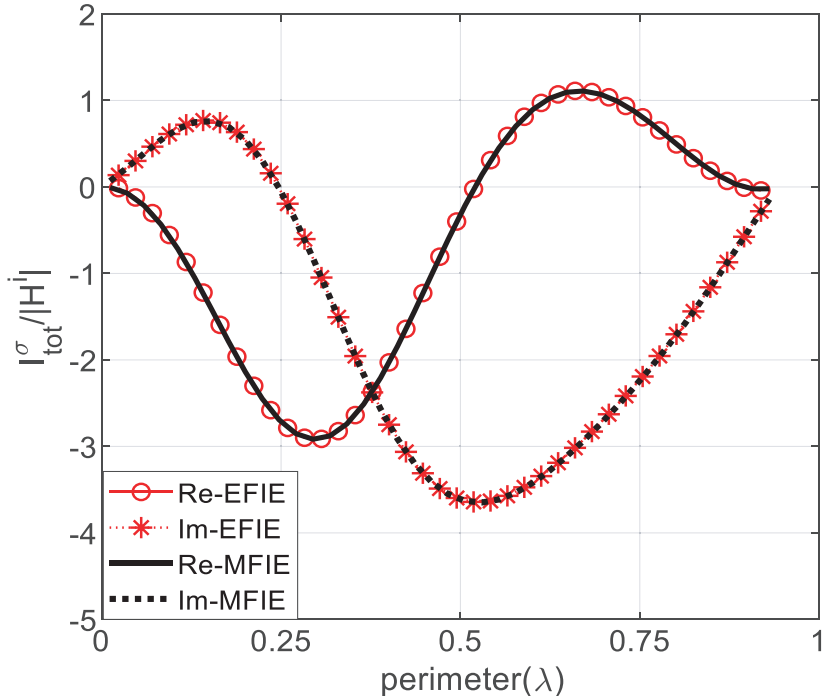

(a)

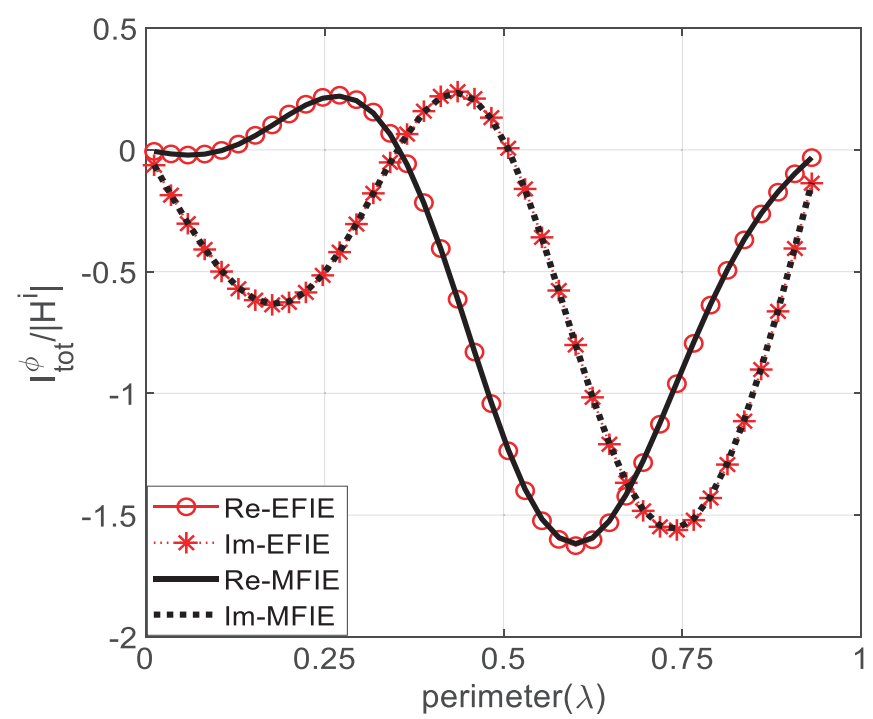

(b)

Figure 7. Corresponding total current, obtained with the MFIE (black) and EFIE (red). The incidentfield parameters and the geometry of the sphere are the same as in Figs. 5 and 6. (a) Corresponding total current in tangential direction in the plane $\phi=0$. (b) Corresponding total current in the $\phi$ direction in the plane $\phi=\pi / 2$.

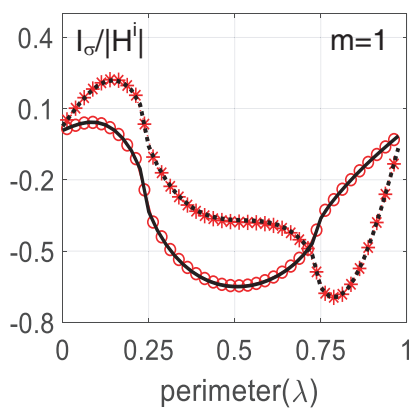

(a)

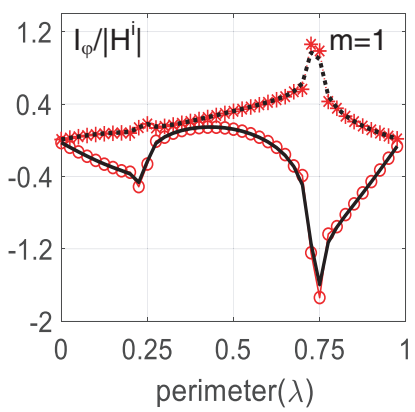

(b)

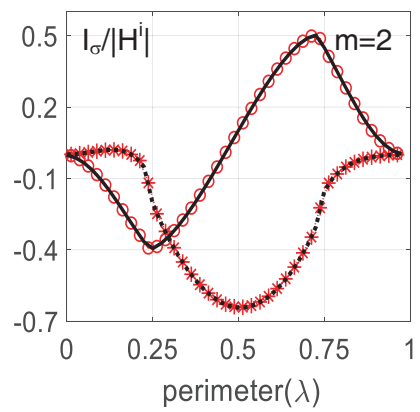

(c)

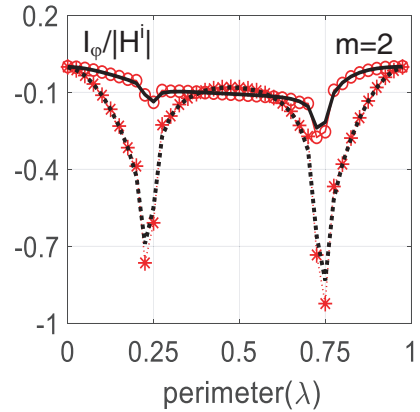

(d)

- - Re-EFIE $\cdots *$ *..Im-EFIE —Re-MFIE $\cdots \cdot . .$. Im-MFIE

Figure 8. Surface currents obtained from the MFIE (black), compared with the ones of the EFIE (red). The results are reported for a perfectly conducting cylinder with radius and height of $r=0.25 \lambda$, and $h=0.5 \lambda$, for a $\theta$-polarized incident field. (a), (b) Tangential and $\phi$-directed current for the mode $m=1$. (c), (d) Same as (a) and (b) except that the results are reported for mode index $m=2$.

as opposed to the singularity extraction approach, we computed the MGF integral purely numerically based on the Gaussian quadrature method and at the singularity, i.e., when the source and observation points coincide, the MGF is replaced by zero. By comparing Fig. 9 and Fig. 11, it can be clearly observed that the current density exhibits much more overshoot than the EFIE result in case the singularity is not extracted and properly handled. So far, we have verified the accuracy of the proposed method for several representative lower and higher-order modes. It is however not yet clear how the total currents calculated from the MFIE and EFIE compare to each other, which would demonstrate the accuracy for all modes. By employing Eq. (4), one can readily obtain the associated total currents, which are plotted in Figs. 12(a) and (b). In particular, the maximum relative deviation between the EFIE and MFIE methods was calculated to be $7.5 E-3$ and $8.5 E-2$ for $I_{\text {tot }}^{\sigma}$ and $I_{\text {tot }}^{\phi}$, respectively. For further assessment of the proposed method, we investigate the performance of the method for a well-known case in the literature $[22,36]$, which is the scattering from a PEC circular cylinder with radius $2 \lambda$ and 


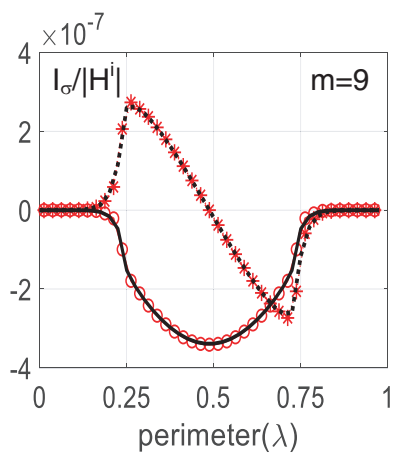

(a)

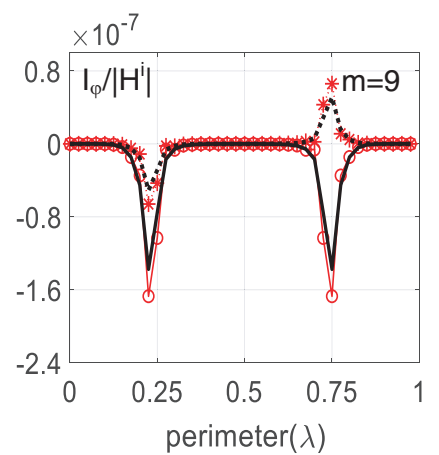

(b)

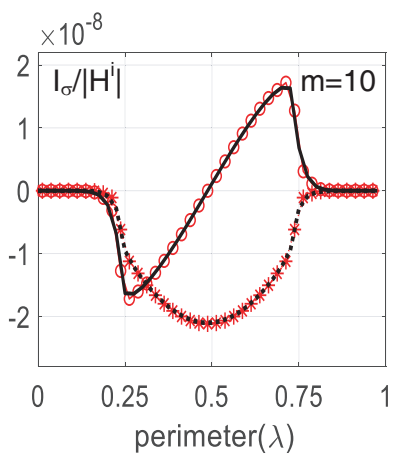

(c)

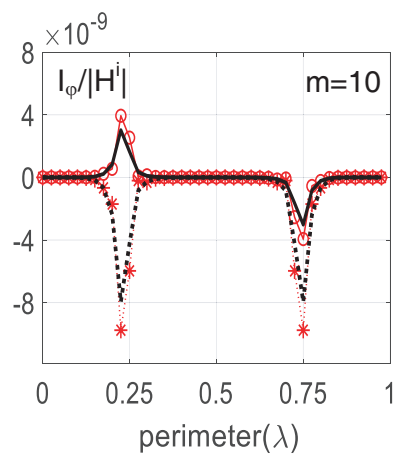

(d)

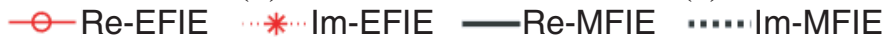

Figure 9. Surface currents obtained from the MFIE (black), compared with the ones of the EFIE (red). The results are reported for a PEC cylinder with height and radius of $r=0.25 \lambda$, and $r=0.5 \lambda$, for a $\theta$-polarized incident field. (a), (b) Tangential and $\phi$-directed current for the mode $m=9$. (c), (d) Same as (a) and (b) except that the results are reported for the mode $m=10$.

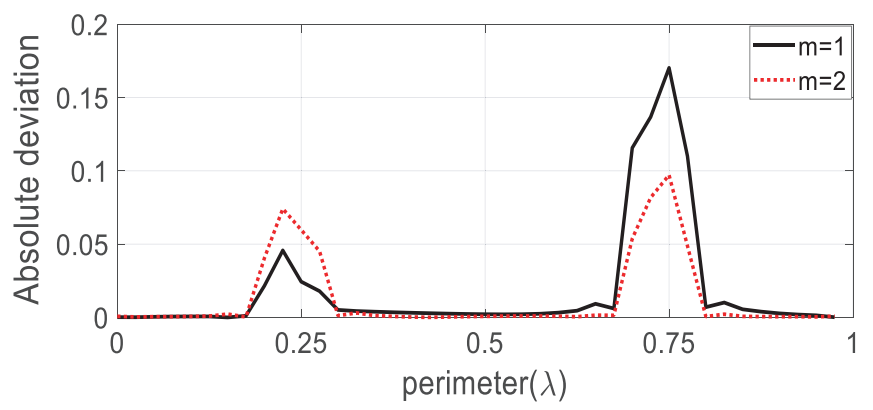

(a)

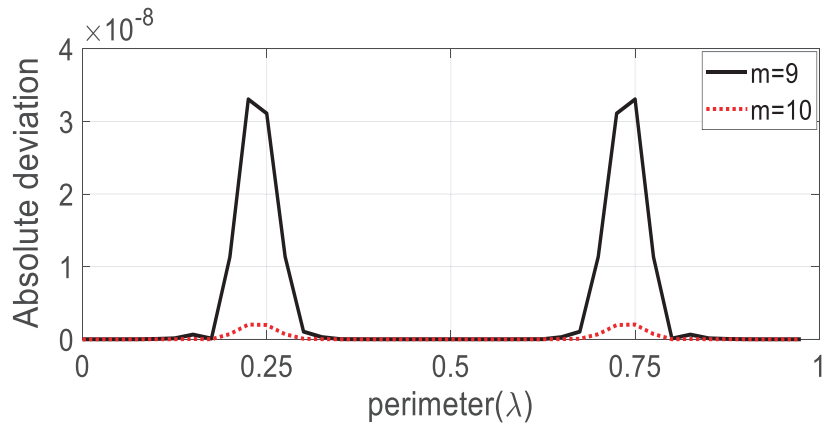

(b)

Figure 10. Absolute deviation between the $\phi$-directed surface currents obtained via the MFIE and EFIE. (a) For modes $m=1$ and $m=2$. (b) For modes $m=9$ and $m=10$.

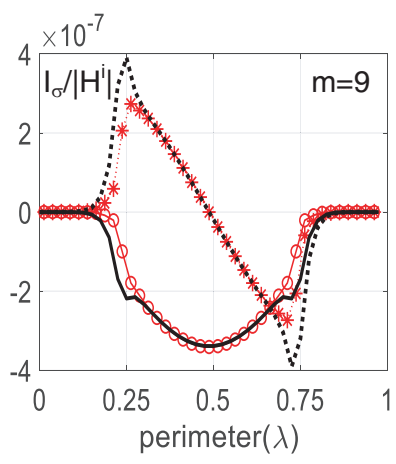

(a)

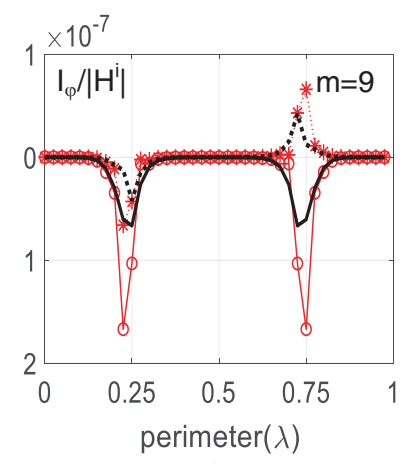

(b)

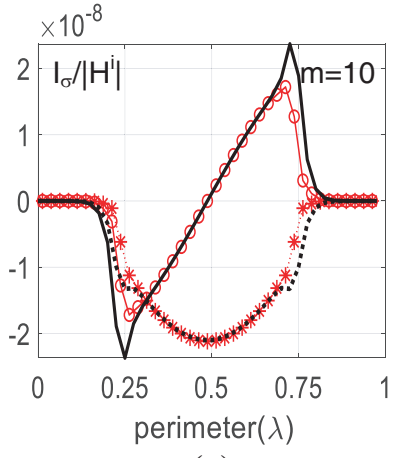

(c)

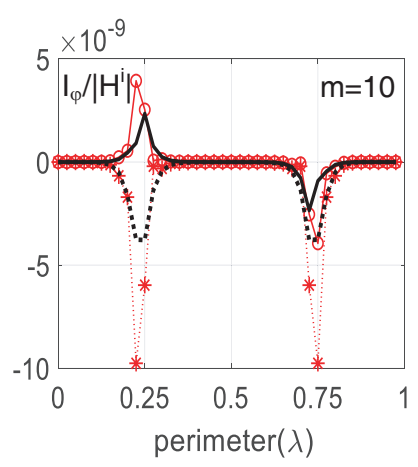

(d)

-Re-EFIE $\cdots *$ *.Im-EFIE —Re-MFIE $\cdots \cdot$...Im-MFIE

Figure 11. Surface currents obtained from the MFIE when no singularity extraction method is applied in the numerical computation (black), compared with the ones of the EFIE (red). The results are reported for a PEC cylinder with height and radius of $r=0.25 \lambda$, and $r=0.5 \lambda$, for a $\theta$-polarized incident field. (a), (b) Tangential and $\phi$-directed current for the mode $m=9$. (c) (d) Same as (a) and (b) except that the results are reported for $m=10$. 
height $2 \lambda$. The incident field in this case is a $\theta$-polarized plane wave with $\theta_{i}=\pi / 4$. Fig. 13(a) indicates the current in the tangential direction in the plane $\phi=0$ and compares it with the data reported in the literature based on the EFIE [22]. The $\phi$-directed current at $\phi=\pi / 2$ is presented in Fig. 13(b). It is observed that the total currents calculated from the proposed MFIE method (black curves in Fig. 13) match the ones obtained from the literature, based on the EFIE, corresponding to a maximum relative error of 0.1 and 0.09 for the tangential and $\phi$-directed currents, respectively.

So far we have focused our attention to the corresponding surface currents. Another good indicator

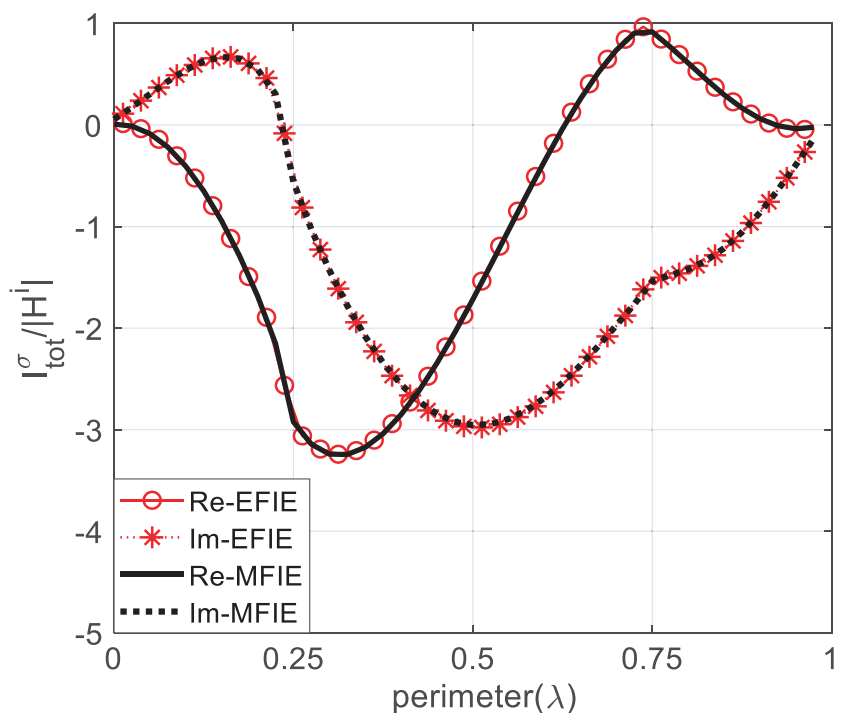

(a)

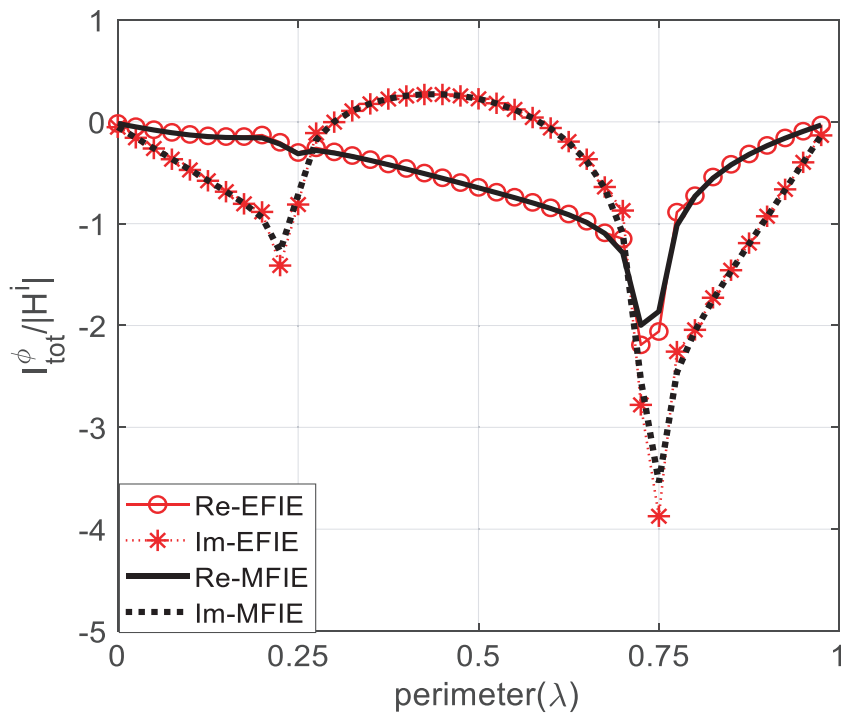

(b)

Figure 12. Corresponding total current, obtained based on the MFIE (black) and EFIE (red). The incident-field parameters and the geometry of the cylinder are the same as Fig. 8 and 9. (a) Total current in the tangential direction in the plane $\phi=0$. (b) Total current in the $\phi$ direction in the plane $\phi=\pi / 2$.

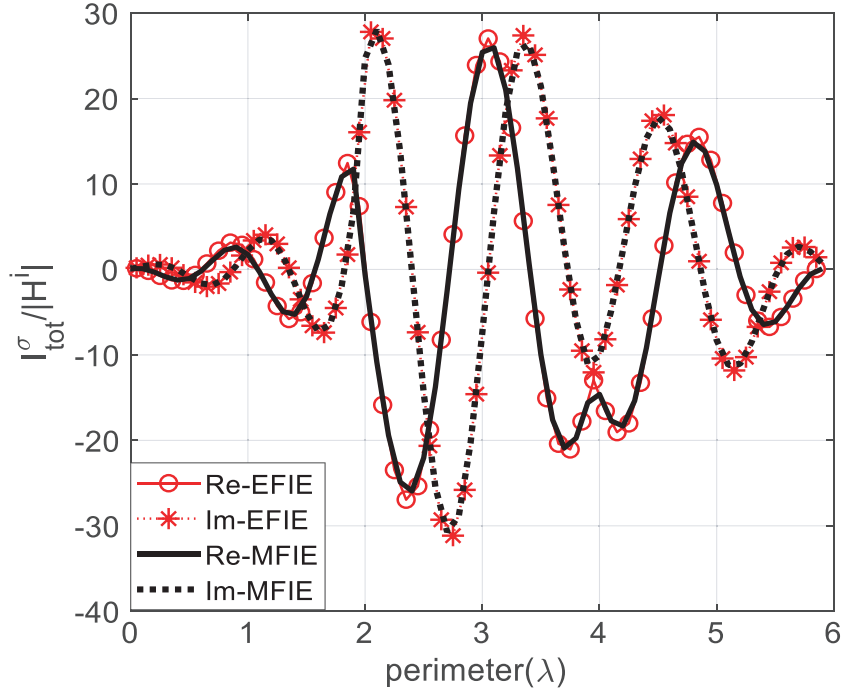

(a)

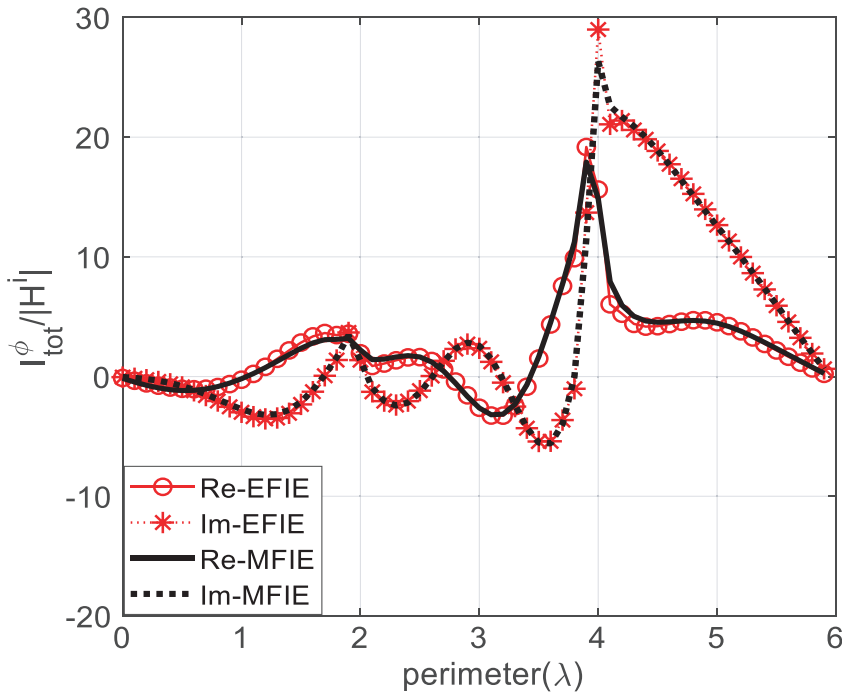

(b)

Figure 13. Comparing the accuracy of our proposed method with the literature $[22,36]$. The figure shows the total current of a PEC cylinder with the radius of $r=2 \lambda$ and the height of $2 \lambda$. (a) Tangential total current in the plane $\phi=0$ (b) $\phi$-directed total current in the plane $\phi=\pi / 2$. 


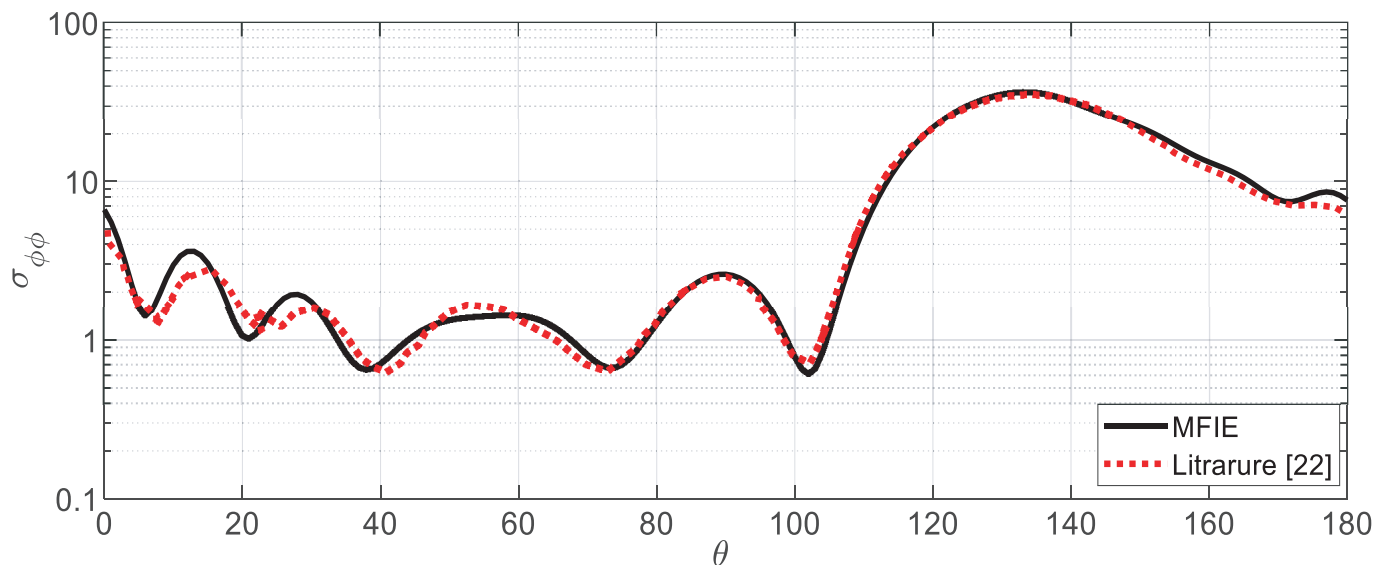

Figure 14. Bi-static RCS component $\sigma_{\phi \phi}$ of the PEC cylinder investigated in Fig. 13. Corresponding $\sigma_{\phi \phi}$ calculated based on the proposed method (solid back curve) and compared to the literature [22] (dotted red curve). The parameters of the incident field and the corresponding observation plane are specified in the text.

by which the correctness of the proposed MFIE method can be verified is the bi-static radar cross-section (RCS). Here, we provide one component of the $2 \times 2$ RCS matrix, namely the co-polarization component $\sigma_{\phi \phi}$ for an incident plane wave polarized in the $\phi$ direction, corresponding to the PEC cylinder studied in Fig. 13. Fig. 14 shows the corresponding $\sigma_{\phi \phi}$ in the observation plane $\phi_{o b s}=0$, for a $\phi$-polarized incident plane wave with the incident angle of $\theta_{i n}=\pi / 4$ and $\phi_{i n}=0$. The results are calculated based on the proposed MFIE technique (solid black curve), and compared to the literature [22] (dotted red curve). The results of this figure provide further evidence for the correctness of the proposed method.

Finally, in Table 1, the maximum relative deviation between the EFIE and MFIE results, as well as the corresponding computation times for solving matrix equations in Eq. (8) for Fourier modes $0 \leq m \leq 15$, has been reported for all the geometries investigated in the paper. It should be noted that, owing to the symmetry of $G_{m}$ with respect to $\pm m$ in Eq. (12), $G_{m}$, and therefore also the matrix elements in Eq. (9), for negative mode indices can be directly obtained from computed ones for the positive mode indices. The computation was performed using MATLAB 2019b on a laptop with 16 GB of RAM and an Intel core i7-8850H processor.

Table 1. Computational characteristics of scattering of PEC BOR with different shapes and dimensions.

\begin{tabular}{|c|c|c|c|c|c|}
\hline Object & $\begin{array}{c}\text { Perimeter of } \\
\text { generating } \\
\text { curve }\end{array}$ & $\begin{array}{c}\text { Corresponding } \\
\text { figure }\end{array}$ & $\begin{array}{c}\text { EFIE method } \\
\text { time }(\mathrm{sec}) \\
0 \leq m \leq 15\end{array}$ & $\begin{array}{c}\text { MFIE method } \\
\text { time }(\mathrm{sec}) \\
0 \leq m \leq 15\end{array}$ & $\begin{array}{c}\text { Max } \\
\text { relative } \\
\text { deviation }\end{array}$ \\
\hline sphere & $0.3 \pi \lambda$ & 6 & 229 & 190 & $0.6 E-2$ \\
cylinder & $\lambda$ & 11 & 234 & 186 & $8.5 E-2$ \\
cylinder & $6 \lambda$ & 12 & 2949 & 2449 & $9.1 E-2$ \\
\hline
\end{tabular}

\section{CONCLUSION}

In conclusion, a method was proposed to characterize the electromagnetic scattering from perfectly conducting bodies of revolution by means of the MFIE. The proposed method is based on efficiently and accurately determining the modal Green function that appears in the MFIE. In the proposed approach, the regular parts of the impedance matrix elements were computed using a fast Fourier transform (FFT), whereas the associated singular parts were evaluated based on two simple recurrence 
relations, thus enabling an efficient computation for all modes of interest in one go. The accuracy of the proposed method was demonstrated by analyzing the scattering from PEC BORs having smooth and non-smooth geometries, and by comparing the results with a similar approach for the EFIE. The proposed method, based on the MFIE, can be combined with its electric-field counterpart, allowing one to get rid of interior resonances that occur when employing either of these integral equations separately.

\section{REFERENCES}

1. Ubeda, E., J. M. Rius, and A. Heldring, "Nonconforming discretization of the electric-field integral equation for closed perfectly conducting objects," IEEE Transactions on Antennas and Propagation, Vol. 62, No. 8, 4171-4186, 2014.

2. Freno, B. A., W. A. Johnson, B. F. Zinser, D. R. Wilton, F. Vipiana, and S. Campione, "Characterization and integration of the singular test integrals in the method-of-moments implementation of the electric-field integral equation," Engineering Analysis with Boundary Elements, Vol. 124, No. 8, 185-193, 2021.

3. Abdelmageed, A., "Efficient evaluation of modal Green's functions arising in EM scattering by bodies of revolution," Progress In Electromagnetics Research, Vol. 27, 337-356, 2000.

4. Mohsen, A. A. K. and A. K. Abdelmageed, "A fast algorithm for treating EM scattering by bodies of revolution," AEU - International Journal of Electronics and Communications, Vol. 55, No. 3, 164-170, 2001.

5. Yu, W. M., D. G. Fang, and T. J. Cui, "Closed form modal Green's functions for accelerated computation of bodies of revolution," IEEE Transactions on Antennas and Propagation, Vol. 56, No. 11, 3452-3461, 2008.

6. Hamed, S. M. A. and S. O. Bashir, "New exact series for modal Green's function," 2015 International Conference on Computing, Control, Networking, Electronics and Embedded Systems Engineering (ICCNEEE), 83-86, 2015.

7. Zubair, M., M. A. Francavilla, D. Zheng, F. Vipiana, and G. Vecchi, "Dual-surface electric field integral equation solution of large complex problems," IEEE Transactions on Antennas and Propagation, Vol. 64, No. 6, 2577-2582, 2016.

8. Bolli, P., G. G. Gentili, R. Nesti, and G. Pelosi, "Coupled BORs scattering via an efficient MoM solution of CFIE," Microwave and Optical Technology Letters, Vol. 37, No. 3, 180-183, 2003.

9. Yla-Oijala, P., "Numerical analysis of combined field integral equation formulations for electromagnetic scattering by dielectric and composite objects," Progress In Electromagnetics Research, Vol. 3, 19-43, 2008.

10. Hodges, R. E. and Y. Rahmat-Samii, "The evaluation of MFIE integrals with the use of vector triangle basis functions," Microwave and Optical Technology Letters, Vol. 14, No. 1, 9-14, 1997.

11. Tong, M. S. and X. J. Huang, "Accurate solution of electromagnetic scattering by super-thin conducting objects based on magnetic field integral equation," IEEE Transactions on Antennas and Propagation, Vol. 65, No. 10, 5633-5638, 2017.

12. Gurel, L. and O. Ergul, "Singularity of the magnetic-field integral equation and its extraction," IEEE Antennas and Wireless Propagation Letters, Vol. 4, No. 1, 229-232, 2005.

13. Ergul, O. and L. Gurel, "Improving the accuracy of the magnetic field integral equation with the linear-linear basis functions," Radio Science, Vol. 41, No. 04, 1-15, 2006.

14. Mohsen, A. A. K. and A. K. Abdelmageed, "Magnetic field integral equation for electromagnetic scattering by conducting bodies of revolution in layered media," Progress In Electromagnetics Research, Vol. 24, No. 3, 19-37, 1999.

15. Andreasen, M., "Scattering from bodies of revolution," IEEE Transactions on Antennas and Propagation, Vol. 13, No. 2, 303-310, 1965.

16. Mautz, J. R. and R. F. Harrington, "Radiation and scattering from bodies of revolution," Applied Scientific Research, Vol. 20, No. 1, 405-435, 1969.

17. Glisson, A. W. and D. R. Wilton, "Simple and efficient numerical techniques for treating bodies of revolution," Mississippi Univ University, 1979. 
18. Wood, W. D., A. W. Wood, and J. L. Fleming, "EM scattering from bodies of revolution using the locally corrected Nyström method," IEEE Antennas and Propagation Society Symposium, Vol. 4, 4036-4039, 2004.

19. Vidal, C. F. V. P. and U. C. Resende, "Solution of integral equation in scattering analysis of conducting bodies of revolution by mom with first type elliptic integrals," Proceedings of the $I V$ International Conference on Computational Methods for Coupled Problems, 1232-1238, 2011.

20. Lai, J. and M. O'Neil, "A fast and high order algorithm for the electromagnetic scattering of axis-symmetric objects," 2018 IEEE International Conference on Computational Electromagnetics (ICCEM), 1-2, 2018.

21. Gedney, S. and R. Mittra, "The use of the FFT for the efficient solution of the problem of electromagnetic scattering by a body of revolution," IEEE Transactions on Antennas and Propagation, 92-95, 1988.

22. Gedney, S. D. and R. Mittra, "The use of the FFT for the efficient solution of the problem of electromagnetic scattering by a body of revolution," IEEE Transactions on Antennas and Propagation, Vol. 38, No. 3, 313-322, 1990.

23. Tong, M. S. and W. C. Chew, "Evaluation of singular Fourier coefficients in solving electromagnetic scattering by body of revolution," Radio Science, Vol. 43, No. 04, 1-9, 2008.

24. Su, T., D. Ding, Z. Fan, and R. Chen, "Efficient analysis of EM scattering from bodies of revolution via the ACA," IEEE Transactions on Antennas and Propagation, Vol. 62, No. 02, 983-985, 2013.

25. Gibson, W. C., The Method of Moments in Electromagnetics, CRC Press, 2014.

26. Schmitz, J. L., "Efficient solution for electromagnetic scattering using the dual-surface magneticfield integral equation for bodies of revolution," Proceedings of IEEE Antennas and Propagation Society International Symposium and URSI National Radio Science Meeting, 2318-2321, 1994.

27. Fleming, J. L., A. W. Wood, and J. W. D. Wood, "Locally corrected Nyström method for EM scattering by bodies of revolution," Journal of Computational Physics, Vol. 196, No. 1, 41-52, 2004.

28. Úbeda Farré, E., Contribution to the Improvement of Integral Equation Methods for Penetrable Scatterers, Universitat Politècnica de Catalunya, 2001.

29. Resende, Ú. C., F. J. S. Moreira, and O. M. C. Pereira-Filho, "Efficient evaluation of singular integral equations in moment method analysis of bodies of revolution," Journal of Microwaves, Optoelectronics and Electromagnetic Applications (JMOe), Vol. 6, No. 2, 373-391, 2007.

30. Vaessen, J. A. H. M., M. C. van Beurden, and A. G. Tijhuis, "Accurate and efficient computation of the modal Green's function arising in the electric-field integral equation for a body of revolution," IEEE Transactions on Antennas and Propagation, Vol. 60, No. 7, 3294-3304, 2012.

31. Trefethen, L. N. and J. Weideman, "The exponentially convergent trapezoidal rule," SIAM Review, Vol. 56, No. 3, 385-458, 2014.

32. Biggs, F., L. B. Mendelsohn, and J. B. Mann, "Hartree-Fock Compton profiles for the elements," Atomic Data and Nuclear Data Tables, Vol. 16, No. 3, 201-309, 1975.

33. Wolfram Research, NIntegrate, Wolfram Language function, https://reference.wolfram.com/ language/ref/NIntegrate.html, 1988 (updated 2014).

34. Umashankar, K. R., "Numerical analysis of electromagnetic wave scattering and interaction based on frequency-domain integral equation and method of moments techniques," Wave Motion, Vol. 10, No. 6, 493-525, 1988.

35. Harrington, R. F., "The method of moments in electromagnetics," Journal of Electromagnetic Waves and Applications, Vol. 1, No. 3, 181-200, 1987.

36. Vaessen, J. A. H. M., Efficient Modeling of Electromagnetic Fields in Stochastic Configurations, Technische Universiteit Eindhoven, 2015. 